\title{
Automotive Configuration Study on Aftermarket
}

\author{
Hongtao Liu
}

\author{
Automotive Engineering institute, Jiangxi University of Technology, Nanchang 330098, China
}

Keywords: Automotive aftermarket; Demand; Configuration model; Product family; Configuration; PDM

\begin{abstract}
With the prosperity of automotive aftermarket and the growth of customers' demand for personalized products, automotive configuration study based on aftermarket research has become inevitable. Focusing on the characteristics of automobile products, the paper studies on the main business activities of automotive design and key technologies systematically and discusses the products configuration modeling, configuration solving, configuration management and other issues, combining business projects with theory to validate the correctness of the model and algorithm. Firstly, the development status of automotive aftermarket system and after-market customer demand is introduced primarily. Based on the need of aftermarket configuration requirements and aftermarket configuration contents, and according to the characteristics of the automotive aftermarket, the paper adopts the rule-based and case-based configuration methods. Finally, the paper combines configuration theory with projects to develop the vehicle configuration system based on aftermarket demand and study the overall framework of the system development, system development platform and system structure and function. The paper also establishes configuration-related customer requirements capture model, automotive configuration module, automotive configuration management model.
\end{abstract}

\section{Introduction}

The society development makes automotive industry change greatly in competition environment and development mode. Currently, under the requirements of realizing customized products in the large-scale customized conditions, the industry faces the development of concerned products whole cycle. The whole product cycle includes products development, production, sale, service and recycle. With the fast development of automotive aftermarket, it gets more and more attentions as a part of automotive products cycles. According to the definition of American Automotive Aftermarket Industry Association, AAIA, the so-called "automotive aftermarket" means the maintenance and repair services after sale and the needed automotive parts, accessory and material market.

The above features of automotive aftermarket development makes automotive manufacturer must face the issue to solve upgrading, maintenance problems. The products configuration research of automotive aftermarket is conducted based on customers' demand and the sold products, which enable automotive manufacturer face the fast changes of aftermarket better: 1)realize connotative diversification of vehicle configuration, which not only includes the technical maintenance, sale, recycle of the parts, but also relates information service, financial service, etc. (2) realize the individualization of vehicle configuration based on automotive aftermarket. Fierce competition and individualized demand force the model development and manufacturing mode must take customers as the center, realizing customized design. Traditional design methods are only suitable for uniform 
and stable market, not for the current market demand. Personalized design shows large advantages. (3) realize agility of vehicle configuration based on automotive aftermarket. To shorten the time to market of new car models and take up positive position, the automotive development must respond the market in time, process various market information and decisions effectively and develop new products fast.

Automotive industry is a competitive technology-intensive industry. In order to adapt to the changing market, various car manufacturing companies emphasize automobile research and development. But the automobile development still exist some shortcomings:

Dynamic characteristics research of product configuration is not enough. In the current research on product configuration, the research focuses on solving aspects of modeling product configuration, knowledge representation and configuration issues. These studies have a common point: they all assume the product configuration model is static, but in fact, the configuration model of the product itself is dynamic evolution, which means that there must be management and configuration maintenance issues on the models evolution and history.

It lacks of concerns on automobile aftermarket. Aftermarket demand feedback for automobile products can improve the configuration design efficiency, but the current study lacks of product configuration process management that based on aftermarket demand. The lack of product configuration versions management and study of product configuration has specific performance on weak support of after sales maintenance. It also lacks of upgrade and maintenance on product version.

It lacks of the focus on the automotive industry supporting areas. The existing automobile product configuration mainly focuses on design research, but lack of considering product life cycle configuration. Product configuration mainly emphasizes on configuring the technical field, and lack of consideration of non-technical areas such as finance, services and so on.

According to automotive vehicle development features of automotive companies, this paper proposes the overall framework of vehicle configuration research and its content based on automobile aftermarket, as well as analyzing automobile configuration features. In line with the functional structure of automobile products, customer demand, maintenance, automobile can be divided into modules, setting up automobile allocation model based on aftermarket. It also describes the automobile based on the models.

\section{Aftermarket system and related technologies of configuration}

Facing fast changeable market demand, automobile manufacturer needs to establish the configuration models and methods that can respond quickly with its own organization. This chapter will introduce the combination process of automobile aftermarket and configuration model, establishing configuration models related with configuration based on the aftermarket, also it will introduce related technologies of automobile configuration development, to propose the configuration methods based on rules and instances through enterprises development conditions analysis.

The product configuration technology based on rules-driven is a method now widely used; its core content is a rule-based reasoning (Rule Based Reasoning-RBR) process. The general principle is to choose the constraints to complete the structure or function matching to translate customer needs into product structure, which will be implemented in two ways: first, establishing mapping rules base on enterprise-defined product model, abstracting configuration of customer demand information as 
product configuration model, and matching with existing product models by mapping rules; the second is to achieve an optional matching based on the enterprise-defined product structure directly, and product structure or parameters variations through rules constraints to configure specific structure products. The main purpose of automobile aftermarket configuration process is to maintain the product and upgrade, so we achieve the configuration with customer needs through mapping rules, and establish the appropriate product platforms.

The configuration based on rules will finish two tasks mainly. Firstly, configure the automobile products using the existing rules, that is derive the need theory and knowledge from the known knowledge related with the problem to be solved and related projects; secondly, control searching process, that is determine the scanning sequence of the rules, to determine the triggered rules under each controlling message, based on the configuration steps of rules:

Establish rule base. The base is used to store the rules related with automobile configuration. The automobile products configuration process based on aftermarket refers to the automobile model construction and version management of configuration units, so the rule base includes the variables rule base, version rule base, effectiveness rule base. The defined format of the rules is shown as following:

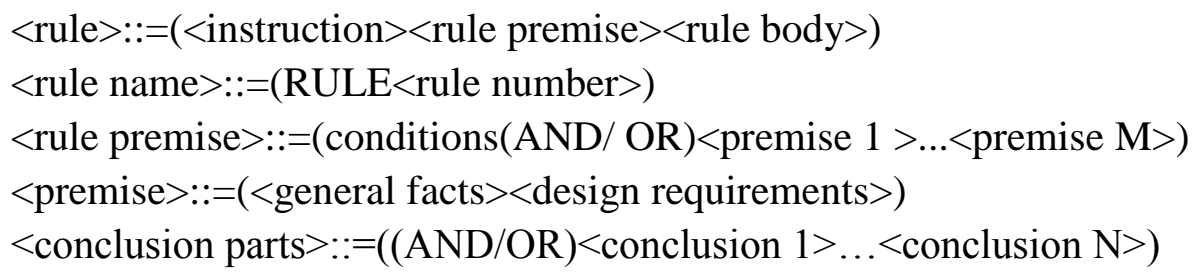

Heuristic search is used for knowledge base and rule base searching. All the searching branches are graded and evaluated according to evaluation function $V(n)$. Selecting the nearest path to target state makes solution and searching fast.

$$
\mathrm{V}(\mathrm{n})=\mathrm{D}(\mathrm{n})+\mathrm{W}(\mathrm{n})
$$

In the function, $\mathrm{D}(\mathrm{n})$ is the depth of node $\mathrm{n} . \mathrm{W}(\mathrm{n})$ is used to calculate the state of current nodes and the distance from target state. $a, b$ are the weight of related functions. The searching process is always directed to the minimum value $\mathrm{V}(\mathrm{n})$ to reach the target state. $\mathrm{W}(\mathrm{n})$ is a heuristic function, to guide convergence of searching process. The solution target of configuration design is to search the optional solution meeting constraint condition. But the best results can only be obtained by finishing the search, which result in unsatisfactory algorithmic efficiency and complexity. In the actual production solution, we can use independent constraint to limit the searching range, to get the best local solution. At the same time, in the searching process, we can adjust searching steps according to the results dynamic regulation. When there are wrong solutions, you must trace back to the branch point of the super level to analyze failure reasons and adjust the searching direction. When the right branch has unreasonable solutions, they must be deleted in time to reduce searching work.

Rules reasoning. Rule-based reasoning is the reasoning technology to make initial evidence and rule base match and reason. Reasoning machine is used to control the whole system. It solves the current problems based on inputting data, that is, the information in database, using certain reasoning strategies and the rules in rule base and send the results to the database. The method of rules inference can generally be divided into: forward reasoning, backward reasoning and hybrid reasoning methods. Only in the automobile configuration process, there is hybrid reasoning, accelerating the rules retrieval process. 
However, rule-driven-based product configuration technologies have common shortcomings: First, lack of flexibility. The configuration rules are customized in accordance with the main structure of the product model without performing hierarchy solution of rules customized logic and rules' matching, therefore, main structure modification is bound to modifying or re-customizing rules and corresponding matching, the inference logic; the second is lack of versatility. There is fixed relationship between the product model and configuration rules. The configuration rules of different product models has different rules methods and tools in customizing, lack of support for the product groups and product configuration.

\section{Automobile configuration model based on aftermarket}

Automobile configuration based on aftermarket $t$ is actually the configuration process of product, and is a configuration according to the customer groups' demand for a sold product, to maintain and upgrade the customer purchased product to maximize the benefits of customers. Therefore, the establishment of product configuration model suitable for automobile aftermarket is an important step towards product configuration management.

After transforming the demand for automobile products, how to establish an effective configuration model is a key point to determine the effective allocation of enterprises. Mass customization is the advanced manufacturing mode of 21 st century. It will combine production and mass customization organically, to meet the individual needs of customers while maintaining low production costs and short delivery lead. Mass customization relies on standardized, modular and serialization components, in which modular part is the main, effective methods of automobile configuration based on aftermarket. However, the current module division is basically from the perspective of functions, which is a simple module partition method, but only from a functional point. It depends on a technician or specialist who is experienced or familiar with the technology of the product, with the subjective opinion to divide, although playing a good effect to some extent, from the aspects of automation, efficiency, objectivity and accuracy, this approach has some limitations.

According to the features analysis $\left(C_{1}, C_{2}, C_{3}, C_{4}\right)$ of products module division aforesaid, assume the four features of $\mathrm{i}$ type automobile configuration $N_{i}$, the corresponding value $V_{i 1}, V_{i 2}, V_{i 3}, V_{i 4}$ can be expressed as:

$$
R_{i}=\left[\begin{array}{ccc}
\mathrm{N}_{\mathrm{i}} & \mathrm{C}_{1} & V_{i 1} \\
& \mathrm{C}_{2} & V_{i 2} \\
& \mathrm{C}_{3} & V_{i 3} \\
& \mathrm{C}_{4} & V_{i 4}
\end{array}\right]=\left[\begin{array}{rrr}
N_{i} & \text { Customized possibility } & V_{i 1} \\
\text { functions of configuration units } & V_{i 2} \\
\text { maintenance amount } & V_{i 3} \\
\text { space position of configuration unit } & V_{i 4}
\end{array}\right]
$$

In module division, it will need certain clustering methods. Products modules division should consider the four feature factors aforesaid, which determines the units positions in division. In clustering analysis(module), math measurement to describe the same degree automobile parts should be established first. Using Minkowski distance, that is: 


$$
\mathrm{d}_{p}\left(R_{i}, R_{j}\right)=\left[\sum_{k=1}^{n}\left|V_{i k}-V_{j k}\right|^{p}\right]^{\frac{1}{p}}, p>0
$$

to measure the automobile configuration units $R_{i}, R_{j}$ close and distant degree in characteristic space. When $\mathrm{p}=1,2$, $\infty$ 时, it will be equal with absolute value, Euclid distance and Chebyshev distance. When using the distance aforesaid, it will use the same dimensional variable. So take module division first, and then conduct standardized process of data, and then calculate the distance.

\section{Automobile configuration process based on aftermarket}

Automobile products configuration units are generally formed by components or modules, and components or modules are further broken down into sub-components or sub-modules, which form a product structure tree. In product configuration, for the same types of products, despite the different needs of customers, the corresponding parts of the structure will be different, but the configuration component or module-level is the same. Component or module-level configuration is based on rule configuration, so retrieval and matching steps of the rules are:

Step 1: read customers' demand information;

Step 2: assume Ci configuration of each configuration unit in product model tree as 0 ;

Step 3: take the configuration units in product structure tree according to the width firstly until all of them are matched, then the configuration based on rules is over;

Step 4: if the configuration mark $\mathrm{Ci}$ is not 0 , turn to step 2;

Step 5: relating and matching according to the demand;

Step 5.1: judge whether to judge all the configuration rules of current vertexes, if yes, turn to step 6 ;

Step 5.2: calculate related expression for this configuration units, take the information of the vertex that related with configuration unit and 1 marking;

Step 5.2: match many semantic conditions as "and", "or" according to the relevance of this configuration units;

Step6: determine the configuration results;

Step 6.1: when the searching is over, it still cannot match, then make this configuration unit $\mathrm{Ci}$ as 2 , which means the unit cannot get configuration result based on rules configuration.

Step 6.2: if matching completely, and the vertex mark $\mathrm{Ci}$ is 1 , it means the configuration based on rules is successful.

During configuration process based on instance, calculate the searched rules that failed using the rules-based configuration method. Its core ideology also uses similar principles to match customer needs with case library instances. Customer demand content includes precise and fuzzy demand information. This paper matches the fuzzy customer needs. To calculate the similarity based on the automobile aftermarket of $\mathrm{A}$ and $\mathrm{B}$, you need to calculate the similarity evaluation function, and the evaluation function is not to determine the similarity by instance description but from the numerical calculation.

Matching algorithm is generally one of the following three methods: numerical, heuristic rule or mixing method of the two. In this paper, it takes the most adjacent matching algorithm as the base, and then calculates the similarity of fixed value and interval values, to solve the problems based on fuzzy information retrieval. 
First, consider from the customer needs information. Since the car structure is complex, high-tech, customer demand for vehicles is from performance that can be realized and expected perspective.

Therefore, based on aftermarket customer demand, automobile design is usually starting from the description of the functions which should be realized and based on certain criteria (such as a car's speed, load, cost, etc.) to complete. These specifications are essentially imprecise, vague. Some values are only within a certain interval range. Currently in the calculation of similar characteristics property, if the two attribute values are expressed with explicit values, there must be evaluation function available. However, in some instances, some features attribute value is within a certain interval range. In the automotive industry, the customer may require car prices $\mathrm{p}$ between $50,000-100,000$, and fuel consumption $\mathrm{c}$ is within $61 / 100 \mathrm{~km}-7$ liters / $100 \mathrm{~km}$. The values of $\mathrm{p}$ and $\mathrm{c}$ in the case library are already determined value, so we need to calculate the value of a certain similarity and a range.

If the similarity between two nonnegative value $a$ and $b$ is defined as:

$$
\operatorname{sim}\left(c,[\alpha, \beta]=\frac{\int_{\alpha}^{\beta} \operatorname{sim}(c, x) d x}{\beta-\alpha}\right.
$$

substitute into the function $\operatorname{sim}(a, b)$, we can get:

$$
\operatorname{sim}=(c,[\alpha, \beta])=\frac{\int\left(1-|x=c| / \max (c, x)^{d x}\right)}{\beta-\alpha}=1-\frac{1}{\beta-\alpha} \bullet \int_{\alpha}^{\beta} \frac{|x-c|}{\max (c, x)} d x
$$

We can utilize standardized similarity matrix to determine the similarity between instances. For the most similar instance, its more detailed information can be obtained from the database as a reference for new design.

\section{Conclusions}

In this paper, Shanghai Huapu Automobile Co., Ltd. Project is the background, then we take automobile market as the starting point for the automobile configuration in-depth study, connecting the automotive aftermarket automobile configuration closely, to improve automobile products quality. Here are the main results and conclusions of the study:

On the basis of analyzing the market, the paper studies the model of automotive vehicle configuration. The traditional configuration of automobile products focuses on the structure and functions more. With the development of aftermarket and focus on product life cycle, automobile configuration is much more abundant. Therefore, the study of automobile configuration models also needs to consider the appropriate knowledge in this regard.

Constructing the rule base and case base of automobile configuration, the paper aims at the market-driven car vehicle configuration. This paper proposes a rule-and-case-based automobile configuration, the rule base and case base is the basis to establish vehicle configuration. The content of rule base has different manifestations according to different classification methods. This paper configuration is to build on the foundation of aftermarket, and therefore, automobile division is in accordance with the module configuration model, the rules are divided into product platform rules, optional parts rule, and spare parts rule. The case library includes content instance, instance structure and instance expressions. 
Realize the configuration method based on the rules and instance, and the rule base and case base based on automobile configuration, the paper studies the automobile configuration process of aftermarket-driven rules-based and instance-based. In respect of the semantic-based configuration retrieval method, firstly, rule-based configuration retrieval is performed, then selecting the product configurable item rule, and then selecting spare arts rule, then after retrieving matching rules, then conduct the rules one by one. Rules analysis can be combined by logic and, logic or.

Automobile configuration management based aftermarket, and the configuration based on aftermarket not only need to configure the product configuration, but also need to maintain the configured products. Because the automobile product configuration based on after-market is a complex, dynamic, large-scale system process, the configuration management is very important.

\section{Acknowledgements}

This work was financially supported by the key subject building project (vehicle engineering) of Jiangxi University of Technology.

\section{References}

[1] Tang Wencheng, Yi Hong, Xing Yan, Xiao Jinping. Product Configuration Management Facing Agile Manufacturing. Computer Integrated Manufacturing Systems

[2] Ma Yuan. Product Demand Management Research and Implementation Supporting Mass Customization. Hangzhou: Zhejiang University, master's degree thesis

[3] Yang Qin, Han Jianghong, Zhu Jiacheng. Expression Model of the Individual Product Needs. Modular Machine Tool \& Automatic Manufacturing Technique.

[4] Guo Wei, Ou Yang Xiaohui, Wang Fengqi. Study based on product data management design process analysis model. computer integration manufacturing system.

[5] Guo Wei, Wang Fengqi, Yu Hongshan et al. Research and development of products demand modeling auxiliary tools system. Computer auxiliary and graphic journal.

[6] Wang Fengqi, Yu Hongshan et al. Study on achieving product cycle demand and expanding technology. computer integration manufacturing system.

[7] Chen Yongliang et al. study on achieving product cycle demand and expanding technology facing CE. High-tech communication.

[8] Du Yuming et al. study on products cycle needs analysis and mapping methods. Mechanical engineering journal.

[9] Hu Sumei, Wang Fengqi et al. study on the methods to achieve products needs facing concurrent design. Mechanical design.

[10] Yue Tongqi, Wang Jijun, Wang Haijun et al. intelligent customer demand achieving system structure design. Dalian University Journal. 